\title{
On the limit behaviour of second order relative spectra of self-adjoint operators
}

\author{
Eugene Shargorodsky
}

\begin{abstract}
It is well known that the standard projection methods allow one to recover the whole spectrum of a bounded self-adjoint operator but they often lead to spectral pollution, i.e. to spurious eigenvalues lying in the gaps of the essential spectrum. Methods using second order relative spectra are free from spectral pollution, but they have not been proven to approximate the whole spectrum. L. Boulton ([3] and [4]) has shown that second order relative spectra approximate all isolated eigenvalues of finite multiplicity. The main result of the present paper is that second order relative spectra do not in general approximate the whole of the essential spectrum of a bounded self-adjoint operator.
\end{abstract}

Mathematics Subject Classification (2010). Primary 47A10, 47A58, 47B15; Secondary $65 \mathrm{~F} 15$.

Keywords. Self-adjoint operators, second order relative spectra, projection methods.

\section{Introduction}

Let $\mathscr{H}$ be a Hilbert space and $\mathscr{B}(\mathscr{H})$ be the space of bounded linear operators on $\mathscr{H}$. Let $\mathscr{L}_{1} \subset \mathscr{L}_{2} \subset \cdots \subset \mathscr{L}_{l} \subset \mathscr{L}_{l+1} \subset \cdots$ be an increasing sequence of finite dimensional linear subspaces of $\mathscr{H}$ such that the corresponding orthogonal projections $P_{l}: \mathscr{H} \rightarrow \mathscr{L}_{l}$ converge strongly to the identity operator $I$. Let $\mathfrak{P}(\mathscr{H})$ be the set of all such sequences of subspaces.

Suppose $T=T^{*} \in \mathscr{B}(\mathscr{H})$ and denote the spectrum of $P_{l} T: \mathscr{L}_{l} \rightarrow \mathscr{L}_{l}$ by $\operatorname{Spec}\left(T, \mathscr{L}_{l}\right)$. Then

$$
\lim _{l \rightarrow \infty} \operatorname{Spec}\left(T, \mathscr{L}_{l}\right) \supseteq \operatorname{Spec}(T),
$$

where "lim" is defined in an appropriate way (see, e.g., [1] or [19]). Unfortunately the left-hand side of (1) may be strictly larger than the right-hand side. This is called spectral pollution (see, e.g., [3], [4], [10], [14], [15], [17], and [19]) which is a well known phenomenon in numerical analysis: spurious "eigenvalues" may appear in the gaps of the essential spectrum of $T$ and as a result $\lim _{l \rightarrow \infty} \operatorname{Spec}\left(T, \mathscr{L}_{l}\right)$ may contain points that do not belong to $\operatorname{Spec}(T)$. 
A possible way of dealing with spectral pollution is based on the notion of second order relative spectra which was introduced by E.B. Davies in [9]:

$$
\operatorname{Spec}_{2}\left(T, \mathscr{L}_{l}\right) \stackrel{\text { def }}{=}\left\{\lambda \in \mathbb{C}: P_{l}(T-\lambda I)^{2}: \mathscr{L}_{l} \rightarrow \mathscr{L}_{l} \text { is not invertible }\right\} .
$$

Although the spectrum of a self-adjoint operator $T$ is a subset of $\mathbb{R}$, the $\operatorname{set}_{\operatorname{Spec}_{2}}\left(T, \mathscr{L}_{l}\right)$ may and usually does contain points from $\mathbb{C} \backslash \mathbb{R}$. Since $T^{*}=T$, it is easy to see that $\operatorname{Spec}_{2}\left(T, \mathscr{L}_{l}\right)$ is symmetric with respect to the real line:

$$
\lambda \in \operatorname{Spec}_{2}\left(T, \mathscr{L}_{l}\right) \Longleftrightarrow \bar{\lambda} \in \operatorname{Spec}_{2}\left(T, \mathscr{L}_{l}\right) .
$$

If $\lambda \in \operatorname{Spec}_{2}\left(T, \mathscr{L}_{l}\right)$ then

$$
\operatorname{Spec}(T) \cap[\operatorname{Re} \lambda-|\operatorname{Im} \lambda|, \operatorname{Re} \lambda+|\operatorname{Im} \lambda|] \neq \emptyset
$$

([14] and [19]; see also [12]). This means that if a point of $\operatorname{Spec}_{2}\left(T, \mathscr{L}_{l}\right)$ is close to the real line, then it is close to $\operatorname{Spec}(T)$, i.e. that, in a sense, second order relative spectra do not pollute.

A natural question, which was first posed in [19] (see also [14] and [20]), is whether $\operatorname{Spec}_{2}\left(T, \mathscr{L}_{l}\right),\left(\mathscr{L}_{l}\right)_{l \in \mathbb{N}} \in \mathfrak{P}(\mathscr{H})$ capture the whole spectrum of $T$, i.e. whether or not

$$
\lim _{l \rightarrow \infty} \operatorname{Spec}_{2}\left(T, \mathscr{L}_{l}\right) \supseteq \operatorname{Spec}(T) .
$$

A partial answer to this question was obtained in [3] and [4]:

$$
\lim _{l \rightarrow \infty} \operatorname{Spec}_{2}\left(T, \mathscr{L}_{l}\right) \supseteq\{\text { isolated eigenvalues of } T \text { of finite multiplicity }\} .
$$

The main result of the present paper is that $\operatorname{Spec}_{2}\left(T, \mathscr{L}_{l}\right)$ do not in general approximate the whole of the essential spectrum $\operatorname{Spec}_{e}(T)$ of $T$. In order to state the result, we need the following notation. Let $d_{H}(F, G)$ denote the Hausdorff distance between two sets $F, G \subset \mathbb{C}$ :

$$
d_{H}(F, G) \stackrel{\text { def }}{=} \max \left\{\sup _{x \in F} \inf _{y \in G}|x-y|, \sup _{y \in G} \inf _{x \in F}|x-y|\right\} .
$$

Let $\Sigma \subset \mathbb{R}$ be a compact set,

$$
\begin{aligned}
m \stackrel{\text { def }}{=} \min \Sigma, M \stackrel{\text { def }}{=} \max \Sigma, \quad[m, M] \backslash \Sigma=\bigcup_{j}\left(m_{j}, M_{j}\right), \\
\left(m_{j}, M_{j}\right) \cap\left(m_{l}, M_{l}\right)=\emptyset \text { if } j \neq l .
\end{aligned}
$$

Define

$$
\mathcal{Q}(\Sigma) \stackrel{\text { def }}{=} B[m, M] \backslash \cup_{j} B\left(m_{j}, M_{j}\right),
$$

where $B\left[c_{1}, c_{2}\right]$ and $B\left(c_{1}, c_{2}\right)$ denote the closed and the open disk with the diameter $\left[c_{1}, c_{2}\right]$. 
Theorem 1.1. Let

$$
-\infty<\rho_{-}^{(1)}<\rho_{+}^{(1)}<\rho_{-}^{(2)}<\rho_{+}^{(2)}<\cdots<\rho_{-}^{(n)}<\rho_{+}^{(n)}<+\infty, \quad n \in \mathbb{N},
$$

and let

$$
F \subseteq \mathcal{Q}\left(\bigcup_{j=1}^{n}\left[\rho_{-}^{(j)}, \rho_{+}^{(j)}\right]\right)
$$

be a compact set symmetric with respect to the real line and such that

$$
F \cap\left(\rho_{-}^{(j)}, \rho_{+}^{(j)}\right) \neq \emptyset, \quad j=1, \ldots, n .
$$

Then there exist $T=T^{*} \in \mathscr{B}(\mathscr{H})$ and $\left(\mathscr{L}_{l}\right) \in \mathfrak{P}(\mathscr{H})$ such that

$$
\operatorname{Spec}(T)=\bigcup_{j=1}^{n}\left[\rho_{-}^{(j)}, \rho_{+}^{(j)}\right]
$$

and

$$
d_{H}\left(\operatorname{Spec}_{2}\left(T, \mathscr{L}_{l}\right), F\right) \longrightarrow 0 \text { as } l \rightarrow+\infty \text {. }
$$

Note that

$$
\bigcup_{\left(\mathscr{L}_{l}\right) \in \mathfrak{P}(\mathscr{H})} \lim _{l \rightarrow+\infty} \operatorname{Spec}_{2}\left(T, \mathscr{L}_{l}\right)=\operatorname{Spec}(T) \cup \mathcal{Q}\left(\operatorname{Spec}_{e}(T)\right),
$$

where $T=T^{*} \in \mathscr{B}(\mathscr{H})$ and where "lim" is defined in an appropriate way ([19]; see also [8]).

Acknowledgements. I am grateful to Michael Strauss and to the anonymous referee for very helpful comments and suggestions.

\section{Auxiliary results}

Proposition 2.1. Let $B, M \in \mathscr{B}(\mathscr{H}), B^{*}=B, M^{*}=M \geq 0$. There exist a Hilbert space $\mathscr{H}_{0} \supseteq \mathscr{H}$ and $T=T^{*} \in \mathscr{B}\left(\mathscr{H}_{0}\right)$ such that $B=\left.P T\right|_{\mathscr{H}}, M=\left.P T^{2}\right|_{\mathscr{H}}$, where $P: \mathscr{H}_{0} \rightarrow H_{\text {is }}$ the orthogonal projection, if and only if

$$
B^{2} \leq M
$$

Proof. Suppose such $\mathscr{H}_{0}$ and $T$ exist. Then

$$
\begin{gathered}
\left(B^{2} x, x\right)=\|B x\|^{2}=\|P T x\|^{2}, \\
(M x, x)=\left(P T^{2} x, x\right)=\left(T^{2} x, x\right)=\|T x\|^{2},
\end{gathered}
$$


for all $x \in \mathscr{H}$. Hence $\left(B^{2} x, x\right) \leq(M x, x)$, for all $x \in \mathscr{H}$, i.e. (4) holds (cf. [18], Appendix).

Suppose now (4) holds. Then $M-B^{2} \geq 0$ has a nonnegative square root $\left(M-B^{2}\right)^{1 / 2}$. Let

$$
\mathscr{H}_{0} \stackrel{\text { def }}{=} \mathscr{H} \bigoplus \mathscr{H},
$$

let

$$
P: \mathscr{H}_{0} \longrightarrow \mathscr{H}
$$

be the projection onto the first component, and let

$$
T \stackrel{\text { def }}{=}\left(\begin{array}{cc}
B & \left(M-B^{2}\right)^{1 / 2} \\
\left(M-B^{2}\right)^{1 / 2} & 0
\end{array}\right): \begin{gathered}
\mathscr{H} \\
\underset{\mathscr{H}}{\oplus} \rightarrow \underset{\mathscr{H}}{\oplus}
\end{gathered}=\begin{gathered}
\mathscr{H} \\
\mathscr{H}
\end{gathered} .
$$

Then $T^{*}=T,\left.P T\right|_{\mathscr{H}}=B$,

$$
T^{2}=\left(\begin{array}{cc}
M & B\left(M-B^{2}\right)^{1 / 2} \\
\left(M-B^{2}\right)^{1 / 2} B & M-B^{2}
\end{array}\right)
$$

and $\left.P T^{2}\right|_{\mathscr{H}}=M$.

Lemma 2.2. For any $\rho_{-}<\rho_{+} \in \mathbb{R}, r \in\left(\rho_{-}, \rho_{+}\right)$and $\delta, \varepsilon>0$ there exist $N \in \mathbb{N}$ and Hermitian matrices $B, R \in \mathbb{C}^{N \times N}$ such that $\|R\|<\varepsilon$, $\operatorname{Spec}(B) \subset\left[\rho_{-}, \rho_{+}\right]$, the distance from any point of $\left[\rho_{-}, \rho_{+}\right]$to $\operatorname{Spec}(B)$ is less than $\delta$, and all roots of the equation

$$
\operatorname{det}\left(\lambda^{2} I-2 \lambda B+B^{2}+R^{2}\right)=0
$$

belong to the vertical interval $\{\lambda \in \mathbb{C}: \operatorname{Re} \lambda=r,|\operatorname{Im} \lambda|<\varepsilon\}$.

Proof. It is sufficient to prove the lemma for $\rho_{ \pm}= \pm \rho, \rho>0$ as the general case can be reduced to this one by dealing with $B-\frac{\rho_{-}+\rho_{+}}{2} I$ instead of $B$.

Let $\varepsilon_{0}$ be a small positive number to be specified later and let $w$ be a conformal mapping of the unit disk onto the ellipse with the axes

$$
[-\rho, \rho]+i \frac{\varepsilon_{0}}{2} \text { and } i\left[0, \varepsilon_{0}\right]
$$

such that $\operatorname{Re} w(0)=r$.

Let $b \in C(\mathbb{T})$ and $a \in C(\mathbb{T})$ be the boundary values of $\operatorname{Re} w$ and $\operatorname{Im} w$ respectively. Then $b(\mathbb{T})=[-\rho, \rho]$ and $a(\mathbb{T})=\left[0, \varepsilon_{0}\right]$.

For any $n \in \mathbb{N}$, the $n \times n$ Toeplitz matrix $T_{n}(b)$ with the symbol $b$ is Hermitian and

$$
\left\|T_{n}(b)\right\| \leq\|T(b)\|=\|b\|_{\infty}=\rho,
$$


where $T(b): l^{2} \rightarrow l^{2}$ is the corresponding Toeplitz operator. Hence

$$
\operatorname{Spec}\left(T_{n}(b)\right) \subset[-\rho, \rho]
$$

It follows from Szegö's theorem (see, e.g., Theorem 5.10 in [2]) that the distance from any point of $[-\rho, \rho]$ to $\operatorname{Spec}\left(T_{N}(b)\right)$ is less than $\delta$ provided $N$ is sufficiently large. Fix such an $N$ and set

$$
B \stackrel{\text { def }}{=} T_{N}(b)
$$

and

$$
A \stackrel{\text { def }}{=} \sqrt{2 \rho \varepsilon_{0}} I+T_{N}(a)=A^{*} .
$$

Since $b+i a$ is the boundary value of the function $w$ analytic in the unit disk, $B+i A=i \sqrt{2 \rho \varepsilon_{0}} I+T_{N}(b+i a)$ is a lower triangular matrix with the diagonal entries equal to $i \sqrt{2 \rho \varepsilon_{0}}+b_{0}+i a_{0}$, where

$$
b_{0} \stackrel{\text { def }}{=} \frac{1}{2 \pi} \int_{0}^{2 \pi} \operatorname{Re} w\left(e^{i t}\right) d t=\operatorname{Re} w(0)=r,
$$

and

$$
a_{0} \stackrel{\text { def }}{=} \frac{1}{2 \pi} \int_{0}^{2 \pi} \operatorname{Im} w\left(e^{i t}\right) d t=\operatorname{Im} w(0) \in\left(0, \varepsilon_{0}\right) .
$$

Hence

$$
\operatorname{Spec}(B+i A)=\left\{r+i\left(\sqrt{2 \rho \varepsilon_{0}}+a_{0}\right)\right\},
$$

and

$$
\operatorname{Spec}(B-i A)=\operatorname{Spec}\left((B+i A)^{*}\right)=\left\{r-i\left(\sqrt{2 \rho \varepsilon_{0}}+a_{0}\right)\right\} .
$$

Consider the matrix polynomial

$$
(\lambda I-(B+i A))(\lambda I-(B-i A))=\lambda^{2} I-2 \lambda B+B^{2}-i[B, A]+A^{2},
$$

where the square brackets denote the commutator. The Hermitian matrix

$$
-i[B, A]+A^{2}
$$

is nonnegative. Indeed,

$$
i[B, A]=i\left[B, T_{N}(a)\right] \leq\left(2\|B\|\left\|T_{N}(a)\right\|\right) I \leq 2 \rho \varepsilon_{0} I \leq A^{2},
$$

where the last inequality follows from the non-negativity of the Toeplitz matrix $T_{N}(a)$ with the symbol $a \geq 0$. 
Let $R$ be the nonnegative square root of $-i[B, A]+A^{2}$. Then

$$
\begin{aligned}
\operatorname{det}\left(\lambda^{2} I-2 \lambda B+B^{2}+R^{2}\right) & =\operatorname{det}((\lambda I-(B+i A))(\lambda I-(B-i A))) \\
& =\operatorname{det}((\lambda I-(B+i A))) \operatorname{det}((\lambda I-(B-i A))) .
\end{aligned}
$$

Hence it follows from (7) that all roots of (6) belong to the interval $\{\lambda \in \mathbb{C}: \operatorname{Re} \lambda=$ $r,|\operatorname{Im} \lambda|<\varepsilon$, provided $\sqrt{2 \rho \varepsilon_{0}}+\varepsilon_{0}<\varepsilon$.

It remains to estimate the norm of $R$ :

$$
\begin{aligned}
\|R x\|^{2}=\left(R^{2} x, x\right) & =\left(\left(-i[B, A]+A^{2}\right) x, x\right) \leq 2\|B\|\left\|T_{N}(a)\right\|+\|A\|^{2} \\
& \leq 2 \rho \varepsilon_{0}+\left(\sqrt{2 \rho \varepsilon_{0}}+\varepsilon_{0}\right)^{2},
\end{aligned}
$$

for all $x \in \mathbb{C}^{N}$ with $\|x\|=1$. Choosing $\varepsilon_{0}>0$ such that the right-hand side is less than $\varepsilon^{2}$ we get $\|R\|<\varepsilon$.

Remark 2.3. Let

$$
T \stackrel{\text { def }}{=}\left(\begin{array}{ll}
B & R \\
R & 0
\end{array}\right): \mathbb{C}^{2 N} \longrightarrow \mathbb{C}^{2 N} .
$$

Then the set of the roots of (6) is equal to $\operatorname{Spec}_{2}\left(T, \mathbb{C}^{N}\right)$. Since

$$
\left\|T-\left(\begin{array}{ll}
B & 0 \\
0 & 0
\end{array}\right)\right\|=\|R\|<\varepsilon,
$$

$\operatorname{Spec}(T) \subset\left[\rho_{-}-\varepsilon, \rho_{+}+\varepsilon\right]$ (see, e.g., Theorem V.4.10 in [13]).

Lemma 2.4. Let $\varrho_{-}, \varrho_{+} \in \mathbb{R}$ and let $T \in \mathbb{C}^{n \times n}$ be a Hermitian matrix such that $\operatorname{Spec}(T) \subset\left[\varrho_{-}, \varrho_{+}\right]$. Then for any $\rho_{-}<\varrho_{-}$, any $\rho_{+}>\varrho_{+}$and any $r \in\left(\rho_{-}, \rho_{+}\right)$, $\delta, \varepsilon>0$, one can choose $N$ and $B$ in Lemma 2.2 in such a way that $N \geq 2 n$ and

$$
B=\left(\begin{array}{cc}
T & S \\
S^{*} & K
\end{array}\right)
$$

with $\|S\|_{\mathbb{C}^{N-n} \rightarrow \mathbb{C}^{n}}<\delta$.

Proof. Let $\mu_{1}, \ldots, \mu_{n}$ be the eigenvalues of $T$ repeated according to their multiplicities and let $N \geq 2 n, B^{\prime}, R^{\prime}$ satisfy the conditions in Lemma 2.2 with $\delta_{0} /(2 n)$ in place of $\delta$, where $\delta_{0}=\min \left\{\delta, \varrho_{-}-\rho_{-}, \rho_{+}-\varrho_{+}\right\}$. The distance between any two consecutive distinct eigenvalues of $B^{\prime}$ is less than $\delta_{0} / n$ as otherwise the distance from the centre of the interval between the eigenvalues to $\operatorname{Spec}\left(B^{\prime}\right)$ would have been greater than or equal to $\delta_{0} /(2 n)$. Since the multiplicity of each $\mu_{k}$ is at most $n$, there exist distinct eigenvalues of $B^{\prime}$ which we denote by $\lambda_{ \pm k}, k=1, \ldots, n$ and which satisfy the conditions

$$
\lambda_{-k} \leq \mu_{k} \leq \lambda_{k} \quad \text { and } \quad \lambda_{k}-\lambda_{-k}<2 \delta .
$$


Then there exist $t_{k} \in[0,1]$ such that $\mu_{k}=\left(1-t_{k}\right) \lambda_{-k}+t_{k} \lambda_{k}$. Let $u_{m} \in \mathbb{C}^{N}$, $m= \pm 1, \ldots, \pm n$ be a normalized eigenvector of $B^{\prime}$ corresponding to $\lambda_{m}$ and set

$$
v_{k} \stackrel{\text { def }}{=} \sqrt{1-t_{k}} u_{-k}+\sqrt{t_{k}} u_{k} \text { and } v_{-k} \stackrel{\text { def }}{=}-\sqrt{t_{k}} u_{-k}+\sqrt{1-t_{k}} u_{k} .
$$

Since $\left\{u_{ \pm k}\right\}_{k=1}^{n}$ is an orthonormal set, $\left\|v_{k}\right\|=1=\left\|v_{-k}\right\|$,

$$
\left(v_{k}, v_{-k}\right)=-\sqrt{1-t_{k}} \sqrt{t_{k}}+\sqrt{t_{k}} \sqrt{1-t_{k}}=0,
$$

and $\left(v_{m}, v_{j}\right)=0$ if $m, j= \pm 1, \ldots, \pm n, m \neq \pm j$. Hence $\left\{v_{ \pm k}\right\}_{k=1}^{n}$ is an orthonormal set. Further,

$$
\begin{aligned}
\left(B^{\prime} v_{k}, v_{k}\right) & =\left(\sqrt{1-t_{k}} \lambda_{-k} u_{-k}+\sqrt{t_{k}} \lambda_{k} u_{k}, \sqrt{1-t_{k}} u_{-k}+\sqrt{t_{k}} u_{k}\right) \\
& =\left(1-t_{k}\right) \lambda_{-k}+t_{k} \lambda_{k}=\mu_{k}, \\
\left(B^{\prime} v_{k}, v_{-k}\right) & =\left(\sqrt{1-t_{k}} \lambda_{-k} u_{-k}+\sqrt{t_{k}} \lambda_{k} u_{k},-\sqrt{t_{k}} u_{-k}+\sqrt{1-t_{k}} u_{k}\right) \\
& =\left(\lambda_{k}-\lambda_{-k}\right) \sqrt{1-t_{k}} \sqrt{t_{k}} \in[0, \delta),
\end{aligned}
$$

since $0 \leq \sqrt{1-t_{k}} \sqrt{t_{k}} \leq 1 / 2$. It is also clear that

$$
\left(B^{\prime} v_{k}, v_{m}\right)=0, \quad m \neq \pm k .
$$

Let $U \in \mathbb{C}^{N \times N}$ be a unitary matrix such that

$$
U(\underbrace{0, \ldots, 0,1}_{k}, 0, \ldots, 0)^{T}= \begin{cases}v_{k}, & k=1, \ldots, n, \\ v_{n-k}, & k=n+1, \ldots, 2 n .\end{cases}
$$

Then

$$
U^{*} B^{\prime} U=\left(\begin{array}{cc}
\operatorname{diag}\left\{\mu_{1}, \ldots, \mu_{n}\right\} & S^{\prime} \\
\left(S^{\prime}\right)^{*} & K
\end{array}\right),
$$

where $S^{\prime}=\left(s_{k j}\right)_{n \times(N-n)},\left|s_{k j}\right|<\delta$ if $j=n+k, s_{k j}=0$ if $j \neq n+k, k=$ $1, \ldots, n, j=n+1, \ldots, N$ (see (8) and (9)). It is easy to see that $\left\|S^{\prime}\right\|_{\mathbb{C}^{N-n} \rightarrow \mathbb{C}^{n}}<\delta$.

Let $U_{0} \in \mathbb{C}^{n \times n}$ be a unitary matrix such that

$$
U_{0} T U_{0}^{*}=\operatorname{diag}\left\{\mu_{1}, \ldots, \mu_{n}\right\},
$$

i.e.

$$
U_{0}^{*} \operatorname{diag}\left\{\mu_{1}, \ldots, \mu_{n}\right\} U_{0}=T,
$$

and let

$$
U_{1} \stackrel{\text { def }}{=}\left(\begin{array}{cc}
U_{0} & 0 \\
0 & I_{N-n}
\end{array}\right) \text {. }
$$


Then $U_{1}$ is a unitary matrix and

$$
U_{1}^{*} U^{*} B^{\prime} U U_{1}=\left(\begin{array}{cc}
U_{0}^{*} \operatorname{diag}\left\{\mu_{1}, \ldots, \mu_{n}\right\} U_{0} & U_{0}^{*} S^{\prime} \\
\left(S^{\prime}\right)^{*} U_{0} & K
\end{array}\right)=\left(\begin{array}{cc}
T & S \\
S^{*} & K
\end{array}\right),
$$

where $S \stackrel{\text { def }}{=} U_{0}^{*} S^{\prime}$. It is clear that $\|S\|_{\mathbb{C}^{N-n} \rightarrow \mathbb{C}^{n}}=\left\|S^{\prime}\right\|_{\mathbb{C}^{N-n} \rightarrow \mathbb{C}^{n}}<\delta$.

Let

$$
B \stackrel{\text { def }}{=} V^{*} B^{\prime} V, \quad R \stackrel{\text { def }}{=} V^{*} R^{\prime} V,
$$

where $V \stackrel{\text { def }}{=} U U_{1}$ is a unitary matrix. Then $B^{*}=B, R^{*}=R, \operatorname{Spec}(B)=\operatorname{Spec}\left(B^{\prime}\right)$, $\|R\|=\left\|R^{\prime}\right\|<\varepsilon$, and all zeros of the polynomial

$$
\begin{aligned}
\operatorname{det}\left(\lambda^{2} I-2 \lambda B+B^{2}+R^{2}\right) & =\operatorname{det}\left(V^{*}\left(\lambda^{2} I-2 \lambda B^{\prime}+\left(B^{\prime}\right)^{2}+\left(R^{\prime}\right)^{2}\right) V\right) \\
& =\operatorname{det}\left(\lambda^{2} I-2 \lambda B^{\prime}+\left(B^{\prime}\right)^{2}+\left(R^{\prime}\right)^{2}\right)
\end{aligned}
$$

belong to the interval $\{\lambda \in \mathbb{C}: \operatorname{Re} \lambda=r,|\operatorname{Im} \lambda|<\varepsilon\}$.

Let $T \in \mathbb{C}^{n \times n}$ and $m \leq n, m \in \mathbb{N}$. Then for any $\varepsilon>0$ there exists $\Delta(T, m, \varepsilon)>0$ such that for any $D \in \mathbb{C}^{m \times m}$ with $\|D\|<\Delta(T, m, \varepsilon)$ the Hausdorff distance between the set of roots of the equation

$$
\operatorname{det}\left(\left.P_{m}(\lambda I-T)^{2}\right|_{\mathbb{C}^{m}}+D\right)=0
$$

and $\operatorname{Spec}_{2}\left(T, \mathbb{C}^{m}\right)$ is less than $\varepsilon$ (see, e.g., Theorem 4.10c in [11]).

We will use the following notation

$$
\ell^{2}(N) \stackrel{\text { def }}{=}\left\{x=\left(x_{k}\right)_{k \in \mathbb{N}} \in \ell^{2}: x_{k}=0, k>N\right\} \cong \mathbb{C}^{N}
$$

and will identify vectors $\left(x_{1}, \ldots, x_{N}\right) \in \mathbb{C}^{N}$ with

$$
\left(x_{1}, \ldots, x_{N}, 0,0, \ldots\right) \in \ell^{2}(N) \subset \ell^{2} .
$$

Lemma 2.5. For any $\rho_{-}<\rho_{+} \in \mathbb{R}, r \in\left(\rho_{-}, \rho_{+}\right)$and any sequence $\alpha_{l} \in(0,1)$, $l \in \mathbb{N}$ converging to 0 there exist a self-adjoint operator $T \in \mathscr{B}\left(\ell^{2}\right)$ and $N_{l} \in \mathbb{N}$, $l \in \mathbb{N}$ such that $\operatorname{Spec}(T)=\left[\rho_{-}, \rho_{+}\right], N_{l} \uparrow+\infty$ as $l \uparrow+\infty$, and

$$
\operatorname{Spec}_{2}\left(T, \ell^{2}\left(N_{l}\right)\right) \subset\left\{\lambda \in \mathbb{C}:|\lambda-r|<\alpha_{l}\right\}, \quad l \in \mathbb{N} .
$$

Proof. Similarly to the proof of Lemma 2.2 we can assume that $\left[\rho_{-}, \rho_{+}\right]=[-2,2]$ as the general case can be reduced to this one by dealing with

$$
\frac{4}{\rho_{+}-\rho_{-}}\left(T-\frac{\rho_{-}+\rho_{+}}{2} I\right)
$$

instead of $T$. 
Let $\rho_{0}=\varrho_{0}=0, \alpha_{0}=\delta_{0}=\varepsilon_{0}=1 / 4, N_{0}=1, B_{0}=0$, and $T_{0}=$ $\left(\begin{array}{ll}0 & 0 \\ 0 & 0\end{array}\right)_{2 \times 2}$. The main idea of the proof is to use Lemmas 2.2 and 2.4 and Remark 2.3 to successively construct matrices

$$
\begin{array}{ll}
\ldots \longrightarrow & T_{l} \\
& \downarrow \\
& B_{l+1}=\left(\begin{array}{cc}
T_{l} & S_{l} \\
S_{l}^{*} & K_{l}
\end{array}\right) \longrightarrow \\
& \begin{array}{l}
T_{l+1} \\
\downarrow
\end{array}
\end{array}
$$

in such a way that the spectra of $T_{l}$ converge to [-2,2], the second order relative spectra of $T_{l}$ converge to $r$, while $S_{l}$ and $R_{l}$ have small norms. A most important feature of the construction is that each of the $T_{l}$ and $B_{l}$ matrices goes into the top left corner of the next one: $\cdots \hookrightarrow T_{l} \hookrightarrow B_{l+1} \hookrightarrow T_{l+1} \hookrightarrow B_{l+2} \hookrightarrow \cdots$. To make the construction work, one needs to choose the numbers $\varepsilon, \delta, \varrho$ and $\rho$ in Lemmas 2.2 and 2.4 at each step in an appropriate way. More precisely, we successively construct $N_{l}, \delta_{l}=\varepsilon_{l}, B_{l}, T_{l}$ such that $B_{l}^{*}=B_{l}: \ell^{2}\left(N_{l}\right) \rightarrow \ell^{2}\left(N_{l}\right), T_{l}^{*}=T_{l}: \ell^{2}\left(2 N_{l}\right) \rightarrow$ $\ell^{2}\left(2 N_{l}\right)$,

$$
T_{l} \stackrel{\text { def }}{=}\left(\begin{array}{cc}
B_{l} & R_{l} \\
R_{l} & 0
\end{array}\right),
$$

$\left\|R_{l}\right\|<\varepsilon_{l}, \operatorname{Spec}\left(B_{l}\right) \subset\left[-\rho_{l}, \rho_{l}\right]$, the distance from any point of $\left[-\rho_{l}, \rho_{l}\right]$ to $\operatorname{Spec}\left(B_{l}\right)$ is less than $\delta_{l}, \rho_{l}=2-2^{-l}$,

$$
\delta_{l}=\varepsilon_{l}<\frac{1}{2} \min \left\{\sqrt{\Delta\left(T_{l-1}, N_{l-1}, \alpha_{l-1} / 2\right)}, \alpha_{l}, \varepsilon_{l-1}\right\},
$$

$$
\begin{aligned}
\operatorname{Spec}_{2}\left(T_{l}, \ell^{2}\left(N_{l}\right)\right) & \subset\left\{\lambda \in \mathbb{C}:|\lambda-r|<\varepsilon_{l}\right\} \\
& \subset\left\{\lambda \in \mathbb{C}:|\lambda-r|<\alpha_{l} / 2\right\}, \\
B_{l+1} & =\left(\begin{array}{cc}
T_{l} & S_{l} \\
S_{l}^{*} & K_{l}
\end{array}\right),
\end{aligned}
$$

$\left\|S_{l}\right\|<\delta_{l+1}$, and $\operatorname{Spec}\left(T_{l}\right) \subset\left[-\left(\rho_{l}+\varepsilon_{l}\right), \rho_{l}+\varepsilon_{l}\right] \subset\left[-\varrho_{l}, \varrho_{l}\right], \varrho_{l}=2-3 \cdot 2^{-l-2}<$ $\rho_{l+1}$. The last inclusion follows from (11) as

$$
\varepsilon_{l}<\frac{\varepsilon_{l-1}}{2}<\cdots<\frac{\varepsilon_{0}}{2^{l}}=2^{-l-2},
$$

and

$$
\rho_{l}+\varepsilon_{l}<2-2^{-l}+2^{-l-2}=2-3 \cdot 2^{-l-2}=\varrho_{l}<2-2^{-l-1}=\rho_{l+1} .
$$

Note that the restriction $\delta_{l}=\varepsilon_{l}<\frac{1}{2} \sqrt{\Delta\left(T_{l-1}, N_{l-1}, \alpha_{l-1} / 2\right)}$ (see (11)) is not used at this stage. It will be need later (see (16) below). 
Since $T_{l}^{*}=T_{l}$ and $\operatorname{Spec}\left(T_{l}\right) \subset\left[-\varrho_{l}, \varrho_{l}\right]$,

$$
\left\|T_{l}\right\| \leq \varrho_{l}=2-3 \cdot 2^{-l-2}<2, \quad l \in \mathbb{N}
$$

Let

$$
\widehat{B}_{l} \stackrel{\text { def }}{=}\left(\begin{array}{cc}
B_{l} & 0 \\
0 & 0
\end{array}\right): \ell^{2} \longrightarrow \ell^{2} \quad \text { and } \quad \widehat{T}_{l} \stackrel{\text { def }}{=}\left(\begin{array}{cc}
T_{l} & 0 \\
0 & 0
\end{array}\right): \ell^{2} \longrightarrow \ell^{2}
$$

Suppose $x \in \ell^{2}\left(2 N_{j}\right), j \leq l,\|x\| \leq 1$. Then

$$
\begin{aligned}
\left\|\widehat{T}_{l+1} x-\widehat{T}_{l} x\right\| & \leq\left\|\widehat{T}_{l+1} x-\widehat{B}_{l+1} x\right\|+\left\|\widehat{B}_{l+1} x-\widehat{T}_{l} x\right\| \\
& =\left\|R_{l+1} x\right\|+\left\|S_{l}^{*} x\right\|<\varepsilon_{l+1}+\delta_{l+1}<2^{-l-3}+2^{-l-3}=2^{-l-2}
\end{aligned}
$$

(see (12)), and therefore

$$
\begin{gathered}
\left\|\widehat{T}_{l+m} x-\widehat{T}_{l} x\right\| \leq \sum_{p=0}^{m-1}\left\|\widehat{T}_{l+p+1} x-\widehat{T}_{l+p} x\right\|<\sum_{p=0}^{m-1} 2^{-l-p-2} \\
=2^{-l-1}-2^{-l-m-1}<2^{-l-1}
\end{gathered}
$$

$m \in \mathbb{N}$. Hence $\left(\widehat{T}_{l} x\right)_{l \in \mathbb{N}}$ is a convergent sequence in $\ell^{2}$ for any $x \in \ell^{2}\left(2 N_{j}\right)$, for all $j \in \mathbb{N}$. Since $\left\|\widehat{T}_{l}\right\|=\left\|T_{l}\right\|<2$, for all $l \in \mathbb{N}$ (see (13)), the sequence $\left(\widehat{T}_{l}\right)_{l \in \mathbb{N}}$ is strongly convergent. Let $T \in \mathcal{B}\left(\ell^{2}\right)$ be its limit. Then $T^{*}=T,\|T\| \leq 2$ and

$$
\left\|T x-\widehat{T}_{l} x\right\| \leq 2^{-l-1}, \quad x \in \ell^{2}\left(2 N_{l}\right),\|x\| \leq 1
$$

Further, $\operatorname{Spec}(T)=[-2,2]$. Indeed, take any $\lambda \in[-2,2]$. The distance from $\lambda$ to $\operatorname{Spec}\left(B_{l}\right)$ is less than $2^{-l}+\delta_{l}=2^{-l}+\varepsilon_{l}<2^{-l}+2^{-l-2}$ (see (12)). Using Theorem V.4.10 in [13] as in Remark 2.3, one can show that the distance from $\lambda$ to $\operatorname{Spec}\left(T_{l}\right)$ is less than $2^{-l}+2^{-l-2}+\varepsilon_{l}<2^{-l}+2^{-l-1}$. Hence there exists an eigenvector $x_{l} \in \ell^{2}\left(2 N_{l}\right)$ of $T_{l}$ such that $\left\|x_{l}\right\|=1$ and $\left\|T_{l} x_{l}-\lambda x_{l}\right\|<2^{-l}+2^{-l-1}$. It follows from (14) that

$$
\left\|T x_{l}-\lambda x_{l}\right\|<2^{-l}+2^{-l-1}+2^{-l-1}=2^{-l+1}, \quad l \in \mathbb{N} .
$$

Therefore, $\lambda \in \operatorname{Spec}(T)$. 
By construction,

$$
\begin{aligned}
T_{l} x & =P_{2 N_{l}} B_{l+1} x \\
& =P_{2 N_{l}} P_{N_{l+1}} T_{l+1} x \\
& =P_{2 N_{l}} T_{l+1} x=P_{2 N_{l}} P_{2 N_{l+1}} T_{l+2} x \\
& =P_{2 N_{l}} T_{l+2} x \\
& =\ldots \\
& =P_{2 N_{l}} T_{l+m} x \\
& =P_{2 N_{l}} \widehat{T}_{l+m} x \\
& =\ldots,
\end{aligned}
$$

for $x \in \ell^{2}\left(2 N_{l}\right)$. So,

$$
\left.P_{2 N_{l}} T\right|_{\ell^{2}\left(2 N_{l}\right)}=T_{l}, \quad l \in \mathbb{N} .
$$

Let us now estimate the difference

$$
\left.P_{2 N_{l}} T^{2}\right|_{\ell^{2}\left(2 N_{l}\right)}-T_{l}^{2} .
$$

Since

$$
T_{l+1}^{2}=\left(\begin{array}{cc}
B_{l+1}^{2}+R_{l+1}^{2} & B_{l+1} R_{l+1} \\
R_{l+1} B_{l+1} & R_{l+1}^{2}
\end{array}\right)
$$

and

$$
B_{l+1}^{2}=\left(\begin{array}{cc}
T_{l}^{2}+S_{l} S_{l}^{*} & T_{l} S_{l}+S_{l} K_{l} \\
S_{l}^{*} T_{l}+K_{l} S_{l}^{*} & S_{l}^{*} S_{l}+K_{l}^{2}
\end{array}\right),
$$

we get

$$
\begin{aligned}
\left\|P_{2 N_{l}} T_{l+1}^{2} x-T_{l}^{2} x\right\| \leq & \left\|P_{2 N_{l}} T_{l+1}^{2} x-P_{2 N_{l}} B_{l+1}^{2} x\right\| \\
& \quad+\left\|P_{2 N_{l}} B_{l+1}^{2} x-T_{l}^{2} x\right\| \\
& =\left\|P_{2 N_{l}} R_{l+1}^{2} x\right\|+\left\|S_{l} S_{l}^{*} x\right\| \\
& <\varepsilon_{l+1}^{2}+\delta_{l+1}^{2} \\
& =2 \varepsilon_{l+1}^{2},
\end{aligned}
$$


for $x \in \ell^{2}\left(2 N_{l}\right)$, and $\|x\| \leq 1$, and therefore (see (12))

$$
\begin{aligned}
\left\|P_{2 N_{l}} T_{l+m}^{2} x-T_{l}^{2} x\right\| & \leq \sum_{p=0}^{m-1}\left\|P_{2 N_{l}} T_{l+p+1}^{2} x-P_{2 N_{l}} T_{l+p}^{2} x\right\| \\
& \leq \sum_{p=0}^{m-1}\left\|P_{2 N_{l+p}} T_{l+p+1}^{2} x-T_{l+p}^{2} x\right\| \\
& <2 \sum_{p=0}^{m-1} \varepsilon_{l+p+1}^{2} \\
& <2 \varepsilon_{l+1}^{2} \sum_{p=0}^{m-1} \frac{1}{2^{2 p}} \\
& =2 \varepsilon_{l+1}^{2} \frac{4}{3}\left(1-\frac{1}{2^{2 m}}\right) \\
& <4 \varepsilon_{l+1}^{2},
\end{aligned}
$$

for $m \in \mathbb{N}$. Hence

$$
\left\|\left.P_{2 N_{l}} T^{2}\right|_{\ell^{2}\left(2 N_{l}\right)}-T_{l}^{2}\right\| \leq 4 \varepsilon_{l+1}^{2}<\Delta\left(T_{l}, N_{l}, \alpha_{l} / 2\right)
$$

(see (11)). Finally,

$$
\begin{aligned}
& \left.P_{N_{l}} T\right|_{\ell^{2}\left(N_{l}\right)}=\left.P_{N_{l}} T_{l}\right|_{\ell^{2}\left(N_{l}\right)}=B_{l} \text { and } \\
& \left\|\left.P_{N_{l}} T^{2}\right|_{\ell^{2}\left(N_{l}\right)}-\left.P_{N_{l}} T_{l}^{2}\right|_{\ell^{2}\left(N_{l}\right)}\right\|<\Delta\left(T_{l}, N_{l}, \alpha_{l} / 2\right) .
\end{aligned}
$$

Since $\operatorname{Spec}_{2}\left(T_{l}, \ell^{2}\left(N_{l}\right)\right) \subset\left\{\lambda \in \mathbb{C}:|\lambda-r|<\alpha_{l} / 2\right\},(10)$ follows from the definition of $\Delta\left(T_{l}, N_{l}, \alpha_{l} / 2\right)$.

Remark 2.6. The argument in the proof of Lemma 2.5 does not change if one adds the requirement

$$
\varepsilon_{l}<\frac{1}{2} \sqrt{\Delta\left(T_{l-1}, 2 N_{l-1}, \alpha_{l-1}\right)}
$$

to (11), although one may get a different sequence of matrices $T_{l}$ and, correspondingly, a different limit operator $T$. For these, one has, additionally to the estimates in the proof of Lemma 2.5, the following inequalities

$$
\left\|\left.P_{2 N_{l}} T^{2}\right|_{\ell^{2}\left(2 N_{l}\right)}-T_{l}^{2}\right\| \leq 4 \varepsilon_{l+1}^{2}<\Delta\left(T_{l}, 2 N_{l}, \alpha_{l}\right)
$$

(see (16)). Since $\operatorname{Spec}_{2}\left(T_{l}, \ell^{2}\left(2 N_{l}\right)\right)=\operatorname{Spec}\left(T_{l}\right)$, it follows from the definition of $\Delta\left(T_{l}, 2 N_{l}, \alpha_{l}\right)$ and from what we know about $\operatorname{Spec}\left(T_{l}\right)$, that $\operatorname{Spec}_{2}\left(T, \ell^{2}\left(2 N_{l}\right)\right)$ lies in an $\alpha_{l}$-neighbourhood of $\left[-\varrho_{l}, \varrho_{l}\right]$ and the distance from any point of $[-2,2]$ to $\operatorname{Spec}_{2}\left(T, \ell^{2}\left(2 N_{l}\right)\right)$ is less than $2^{-l}+2^{-l-1}+\alpha_{l}$. Hence $\operatorname{Spec}_{2}\left(T, \ell^{2}\left(2 N_{l}\right)\right)$ converge to $[-2,2]$ while $\operatorname{Spec}_{2}\left(T, \ell^{2}\left(N_{l}\right)\right)$ converge to $\{r\}$ as $l \rightarrow+\infty$. 


\section{Proof of Theorem 1.1}

Let

$$
r_{j} \in F \cap\left(\rho_{-}^{(j)}, \rho_{+}^{(j)}\right),
$$

for $j=1, \ldots, n$, and let $T^{(j)}$ and $N_{l}^{(j)}, l \in \mathbb{N}$ be the same as in Lemma 2.5 but with $r_{j} \in\left(\rho_{-}^{(j)}, \rho_{+}^{(j)}\right)$ in place of $r \in\left(\rho_{-}, \rho_{+}\right)$. Let $\mathscr{H}_{j}=\ell^{2}, \mathscr{H}=\bigoplus_{j=1}^{n} \mathscr{H}_{j}$ and $T=\operatorname{diag}\left\{T^{(1)}, \ldots, T^{(n)}\right\} \in \mathscr{B}(\mathscr{H})$. It is clear that $T=T^{*}$ and $\operatorname{Spec}(T)=$ $\bigcup_{j=1}^{n}\left[\rho_{-}^{(j)}, \rho_{+}^{(j)}\right]$.

Let $F_{l}$ be a finite subset of the interior of $\mathcal{Q}\left(\bigcup_{j=1}^{n}\left[\rho_{-}^{(j)}, \rho_{+}^{(j)}\right]\right)$ symmetric with respect to the real line and such that

$$
d_{H}\left(F_{l}, F\right)<2^{-l-1},
$$

and let $F_{l} \cap\{\lambda \in \mathbb{C}: \operatorname{Im} \lambda \geq 0\}=\left\{\mu_{1}^{(l)}, \ldots, \mu_{n_{l}}^{(l)}\right\}$. For any $k=1, \ldots, n_{l}$ there exist $\lambda_{1, k}^{(l)}, \lambda_{2, k}^{(l)}, \lambda_{3, k}^{(l)} \in \bigcup_{j=1}^{n}\left(\rho_{-}^{(j)}, \rho_{+}^{(j)}\right)$ such that the convex hull of $\left\{\left(\mu_{k}^{(l)}-\lambda_{m, k}^{(l)}\right)^{2}\right\}_{m=1}^{3}$ contains 0 , i.e. there exists

$$
\begin{aligned}
& t_{1, k}^{(l)}, t_{2, k}^{(l)}, t_{3, k}^{(l)} \in[0,1]: t_{1, k}^{(l)}+t_{2, k}^{(l)}+t_{3, k}^{(l)}=1, \\
& \sum_{m=1}^{3} t_{m, k}^{(l)}\left(\mu_{k}^{(l)}-\lambda_{m, k}^{(l)}\right)^{2}=0
\end{aligned}
$$

(see [19]).

Let $\mathscr{L}_{0}=\{0\}, \tilde{N}_{0}=1$, and suppose we have constructed $\mathscr{L}_{0} \subset \mathscr{L}_{1} \subset \cdots \subset$ $\mathscr{L}_{l-1} \subset \mathscr{H}$ and $\tilde{N}_{0}<\tilde{N}_{1}<\cdots<\tilde{N}_{l-1} \in \mathbb{N}$ such that $\mathscr{L}_{p} \subseteq \bigoplus_{j=1}^{n} \ell^{2}\left(\tilde{N}_{p}\right)$, $p=1, \ldots, l-1$. Let us construct $\mathscr{L}_{l}$ and $\tilde{N}_{l}$. Let $\widehat{N}_{l}^{(j)}$ be the smallest number among $N_{l}^{(j)}<N_{l+1}^{(j)}<N_{l+2}^{(j)}<\ldots$ which is greater than or equal to $\tilde{N}_{l-1}$. Then $\mathscr{L}_{l-1} \subseteq \mathscr{L}_{l}^{0} \stackrel{\text { def }}{=} \bigoplus_{j=1}^{n} \ell^{2}\left(\hat{N}_{l}^{(j)}\right)$ and

$$
d_{H}\left(\operatorname{Spec}_{2}\left(T, \mathscr{L}_{l}^{0}\right),\left\{r_{1}, \ldots, r_{n}\right\}\right)<\alpha_{l} .
$$

Let $E(\cdot)$ be the spectral measure of $T$ and let

$$
W_{m, k}^{(l)} \subset \bigcup_{j=1}^{n}\left(\rho_{-}^{(j)}, \rho_{+}^{(j)}\right)
$$

be the $\varepsilon_{l}^{\prime}$-neighbourhood of $\lambda_{m, k}^{(l)}$, where $\varepsilon_{l}^{\prime}$ is a small positive number to be specified later. Since the subspaces $E\left(W_{m, k}^{(l)}\right) \mathscr{H} \subset \mathscr{H}$ are infinite dimensional, we can choose vectors $u_{m, k}^{(l)} \in E\left(W_{m, k}^{(l)}\right) \mathscr{H}$ such that $\left\|u_{m, k}^{(l)}\right\|=1$ and

$$
u_{m, k}^{(l)} \perp T^{q}\left(\mathscr{L}_{l}^{0}\right) \quad \text { and } \quad u_{m, k}^{(l)} \perp T^{q} u_{m^{\prime}, k^{\prime}}^{(l)},
$$


for $q=0,1,2, \quad m, m^{\prime}=1,2,3, k, k^{\prime}=1, \ldots n_{l}$, and $(m, k) \neq\left(m^{\prime}, k^{\prime}\right)$. Let

$$
v_{k}^{(l)}=\sum_{m=1}^{3} \sqrt{t_{m, k}^{(l)}} u_{m, k}^{(l)}, \quad \mathscr{L}_{l}^{\prime}=\mathscr{L}_{l}^{0} \oplus \operatorname{span}\left\{v_{k}^{(l)}\right\}_{k=1}^{n_{l}},
$$

and let $\mathcal{P}_{l}^{0}: \mathscr{H} \rightarrow \mathscr{L}_{l}^{0}$ and $\mathcal{P}_{l}^{\prime}: \mathscr{H} \rightarrow \mathscr{L}_{l}^{\prime}$ be the corresponding orthogonal projections. Then $\left\|v_{k}^{(l)}\right\|=1$,

$$
v_{k}^{(l)} \perp T^{q}\left(\mathscr{L}_{l}^{0}\right), \quad v_{k}^{(l)} \perp T^{q} v_{k^{\prime}}^{(l)}, \quad q=0,1,2, k, \quad k^{\prime}=1, \ldots n_{l}, \quad k \neq k^{\prime},
$$

and

$$
\begin{aligned}
\left.\mathcal{P}_{l}^{\prime}(\lambda I-T)^{2}\right|_{\mathscr{L}_{l}^{0}} & =\left.\mathcal{P}_{l}^{0}(\lambda I-T)^{2}\right|_{\mathscr{L}_{l}^{0}}, \\
\mathcal{P}_{l}^{\prime}(\lambda I-T)^{2} v_{k}^{(l)} & =\left((\lambda I-T)^{2} v_{k}^{(l)}, v_{k}^{(l)}\right) v_{k}^{(l)} \stackrel{\text { def }}{=} p_{k}^{(l)}(\lambda) v_{k}^{(l)} .
\end{aligned}
$$

Hence $\left.\mathcal{P}_{l}^{\prime}(\lambda I-T)^{2}\right|_{\mathscr{L}_{l}^{\prime}}$ is unitarily equivalent to

$$
\left(\begin{array}{cc}
\left.\mathcal{P}_{l}^{0}(\lambda I-T)^{2}\right|_{\mathscr{L}_{l}^{0}} & 0 \\
0 & \operatorname{diag}\left\{p_{1}^{(l)}(\lambda), \ldots, p_{n_{l}}^{(l)}(\lambda)\right\}
\end{array}\right)
$$

and

$$
\operatorname{Spec}_{2}\left(T, \mathscr{L}_{l}^{\prime}\right)=\operatorname{Spec}_{2}\left(T, \mathscr{L}_{l}^{0}\right) \cup \bigcup_{k=1}^{n_{l}}\left\{\lambda \in \mathbb{C}: p_{k}^{(l)}(\lambda)=0\right\} .
$$

By construction, the coefficients of the quadratic polynomial

$$
p_{k}^{(l)}(\lambda)=\left((\lambda I-T)^{2} v_{k}^{(l)}, v_{k}^{(l)}\right)=\sum_{m=1}^{3} t_{m, k}^{(l)}\left((\lambda I-T)^{2} u_{k}^{(l)}, u_{k}^{(l)}\right)
$$

are real and differ by less than $C \varepsilon_{l}^{\prime}$ from those of

$$
q_{k}^{(l)}(\lambda) \stackrel{\text { def }}{=} \sum_{m=1}^{3} t_{m, k}^{(l)}\left(\lambda-\lambda_{m, k}^{(l)}\right)^{2} .
$$

(It follows from the spectral theorem that one can take

$$
C=2 \max \{1,\|T\|\}
$$

here.) Taking $\varepsilon_{l}^{\prime}$ sufficiently small we can ensure that the zeros of $p_{k}^{(l)}(\lambda)$ differ from those of $q_{k}^{(l)}(\lambda)$ by less than $2^{-l-1}$. According to (18), $\mu_{k}^{(l)}$ and its complex conjugate are the zeros of $q_{k}^{(l)}(\lambda)$. Hence it follows from (17), (19), and (20) that

$$
d_{H}\left(\operatorname{Spec}_{2}\left(T, \mathscr{L}_{l}^{\prime}\right), F\right)<\max \left\{\alpha_{l}, 2^{-l}\right\}
$$


Let $\tilde{N}_{l}>\tilde{N}_{l-1}, \tilde{N}_{l}>\hat{N}_{l}^{(j)}, j=1, \ldots, n, P_{(l)}: \mathscr{H} \rightarrow \bigoplus_{j=1}^{n} \ell^{2}\left(\tilde{N}_{l}\right)$ be the orthogonal projection,

$$
\mathscr{L}_{l}=\mathscr{L}_{l}^{0} \oplus \operatorname{span}\left\{P_{(l)} v_{k}^{(l)}\right\}_{k=1}^{n_{l}}
$$

and let $\mathscr{P}_{l}: \mathscr{H} \rightarrow \mathscr{L}_{l}$ be the corresponding orthogonal projection. $\operatorname{Spec}_{2}\left(T, \mathscr{L}_{l}^{\prime}\right)$ is the set of zeros of the determinant of a matrix representation of $\left.\mathcal{P}_{l}^{\prime}(\lambda I-T)^{2}\right|_{\mathscr{L}_{l}^{\prime}}$ which is a polynomial in $\lambda$. If $\widetilde{N}_{l}$ is large, then $P_{(l)} v_{k}^{(l)}$ is close to $v_{k}^{(l)}$, and the coefficients of the polynomial corresponding to $\mathcal{P}_{l}(\lambda I-T)^{2} \mid \mathscr{L}_{l}$ are close to their counterparts corresponding to $\left.\mathcal{P}_{l}^{\prime}(\lambda I-T)^{2}\right|_{\mathscr{L}_{l}^{\prime}}$. Hence taking $\widetilde{N}_{l}$ sufficiently large we get

$$
d_{H}\left(\operatorname{Spec}_{2}\left(T, \mathscr{L}_{l}\right), F\right)<\max \left\{\alpha_{l}, 2^{-l}\right\}
$$

(see Theorem 4.10c in [11]). Note that $\left(\mathscr{L}_{l}\right) \in \mathfrak{P}(\mathscr{H})$ because $\mathscr{L}_{l} \supset \mathscr{L}_{l}^{0}=$ $\bigoplus_{j=1}^{n} \ell^{2}\left(\widehat{N}_{l}^{(j)}\right)$ and $\hat{N}_{l}^{(j)} \geq N_{l}^{(j)} \rightarrow+\infty$ as $l \rightarrow+\infty, j=1, \ldots n$.

Remark 3.1. $\operatorname{Spec}_{2}\left(T, \mathscr{L}_{l}\right)$ constructed in the above proof converge to $F$. The limit behaviour of a sequence of second order relative spectra of $T$ may be considerably more complicated than that. Let, for example, $F_{0} \subseteq \mathcal{Q}\left(\bigcup_{j=1}^{n}\left[\rho_{-}^{(j)}, \rho_{+}^{(j)}\right]\right)$ be another compact set symmetric with respect to the real line and such that

$$
F_{0} \cap F \cap\left(\rho_{-}^{(j)}, \rho_{+}^{(j)}\right) \neq \emptyset, \quad j=1, \ldots, n .
$$

Acting as in the proof above one can construct a sequence $\left(\mathscr{L}_{0, l}\right)$ similar to $\left(\mathscr{L}_{l}\right)$ and such that

$$
d_{H}\left(\operatorname{Spec}_{2}\left(T, \mathscr{L}_{0, l}\right), F_{0}\right) \longrightarrow 0, \quad \text { as } l \rightarrow+\infty .
$$

Then it is easy to extract subsequences from $\left(\mathscr{L}_{l}\right)$ and to $\left(\mathscr{L}_{0, l}\right)$ and to combine them into a new sequence $\left(\mathcal{M}_{l}\right) \in \mathfrak{P}(\mathscr{H})$ in such a way that

$$
d_{H}\left(\operatorname{Spec}_{2}\left(T, \mathcal{M}_{2 l}\right), F\right) \longrightarrow 0 \quad \text { and } \quad d_{H}\left(\operatorname{Spec}_{2}\left(T, \mathcal{M}_{2 l+1}\right), F_{0}\right) \longrightarrow 0,
$$

as $l \rightarrow+\infty$. One can of course carry out a similar procedure with more than just two limit sets $F$ and $F_{0}$.

\section{Concluding remarks}

The sequence $\left(N_{l}\right)$ in the proof of Lemma 2.5 and $\left(\operatorname{dim} \mathscr{L}_{l}\right)$ in the proof of Theorem 1.1 are very rapidly increasing and it is not clear whether the above results have serious implications for "real life" computations involving second order relative spectra. In all numerical examples studied so far (see, e.g., [3], [4], [5], [6], [7], [8], [14], and [21]), second order relative spectra seemed to approximate the whole spectrum quite well. 
Question 1. Can the phenomenon described by Lemma 2.5 and Theorem 1.1 still happen if one restricts the rate of growth of $\operatorname{dim} \mathscr{L}_{l}$ ?

Note that

$$
\lim _{N \rightarrow+\infty}^{*} \operatorname{Spec}_{2}\left(T, \ell^{2}(N)\right) \cap \mathbb{R}=[-2,2]=\operatorname{Spec}(T)
$$

in Remark 2.6. Here

$$
\begin{array}{r}
\lim _{l \rightarrow+\infty}^{*} G_{l} \stackrel{\text { def }}{=}\left\{z \in \mathbb{C}: \text { there are } l_{m} \in \mathbb{N}, \text { and } z_{l_{m}} \in G_{l_{m}}\right. \text { such that } \\
\left.l_{m} \rightarrow+\infty \text { and } z_{l_{m}} \rightarrow z, \text { as } m \rightarrow+\infty\right\}
\end{array}
$$

for $G_{l} \subset \mathbb{C}$, and $l \in \mathbb{N}$.

It is well known that

$$
\lim _{l \rightarrow+\infty} \operatorname{Spec}\left(T, \mathscr{L}_{l}\right) \supseteq \operatorname{Spec}(T), \quad\left(\mathscr{L}_{l}\right) \in \mathfrak{P}(\mathscr{H}),
$$

where

$$
\lim _{l \rightarrow+\infty} G_{l} \stackrel{\text { def }}{=}\left\{z \in \mathbb{C} \mid \exists z_{l} \in G_{l}: \lim _{l \rightarrow+\infty} z_{l}=z\right\}
$$

(see, e.g., [1] or [19]). It is reasonable therefore to use $\lim _{*}$ when approximating $\operatorname{Spec}(T)$ with the help of $\operatorname{Spec}\left(T, \mathscr{L}_{l}\right)$. On the other hand, the non-pollution result (2) shows it is more natural to use $\lim ^{*}$ when approximating $\operatorname{Spec}(T)$ with the help of $\operatorname{Spec}_{2}\left(T, \mathscr{L}_{l}\right)$.

Another natural question is whether or not one can drop condition (3) in Theorem 1.1.

Question 2. Can the limit set of a sequence of second order relative spectra be disjoint from the (essential) spectrum of $T=T^{*} \in \mathscr{B}(\mathscr{H})$ ?

\section{References}

[1] W. Arveson, $C^{*}$-algebras and numerical linear algebra. J. Functional Analysis 122 (1994), 330-360. MR 1276162 Zbl 0802.46069

[2] A. Böttcher and S.M. Grudsky, Toeplitz matrices, asymptotic linear algebra, and functional analysis. Birkhäuser, Basel, 2000. MR 1772773 Zbl 0969.47022

[3] L. Boulton, Limiting set of second order spectra. Math. Comp. 75 (2006), 1367-1382. MR 2219033 Zbl 1102.47019

[4] L. Boulton, Non-variational approximation of discrete eigenvalues of self-adjoint operators. IMA J. Numer. Anal. 27 (2007), 102-121. MR 2289273 Zbl 1116.65062

[5] L. Boulton and N. Boussaiid, Non-variational computation of the eigenstates of Dirac operators with radially symmetric potentials. LMS J. Comput. Math. 13 (2010), 10-32. MR 2593910 Zbl 1227.65105 
[6] L. Boulton and M. Levitin, On approximation of the eigenvalues of perturbed periodic Schrödinger operators. J. Phys. A, Math. Theor., 40 (2007), 9319-9329. MR 2345295 Zbl 1120.81033

[7] L. Boulton and M. Strauss, Stability of quadratic projection methods. Oper. Matrices 1 (2007), 217-233. MR 2327582 Zbl 1139.65037

[8] L. Boulton and M. Strauss, On the convergence of second-order spectra and multiplicity. Proc. R. Soc. Lond. Ser. A Math. Phys. Eng. Sci. 467 (2011), 264-284. MR 2764683 Zbl 1219.65051

[9] E. B. Davies, Spectral enclosures and complex resonances for general self-adjoint operators. LMS J. Comput. Math. 1 (1998), 42-74. MR 1635727 Zbl 0931.47021

[10] E. B. Davies and M. Plum, Spectral pollution. IMA J. Numer. Anal. 24 (2004), 417-438. MR $2068830 \mathrm{Zbl} 1062.65056$

[11] P. Henrici, Applied and computational complex analysis. Vol. I. Wiley Interscience (John Wiley \& Sons), New York etc., 1974. MR 0372162 Zbl 0635.30001

[12] T. Kato, On the upper and lower bounds of eigenvalues. J. Phys. Soc. Japan 4 (1949), 334-339. MR 0038738

[13] T. Kato, Perturbation theory for linear operators. Grundlehren der mathematischen Wissenschaften. Band 132. Springer-Verlag, Berlin etc., 1966. MR 0203473 Zbl 0148.12601

[14] M. Levitin and E. Shargorodsky, Spectral pollution and second order relative spectra for self-adjoint operators. IMA J. Numer. Anal. 24 (2004), 393-416. MR 2068829 Zbl 1060.65056

[15] A. Pokrzywa, Method of orthogonal projections and approximation of the spectrum of a bounded operator. Stud. Math. 65 (1979), 21-29. MR 0554538 Zbl 0437.47004

[16] A. Pokrzywa, Spectra of operators with fixed imaginary parts. Proc. Amer. Math. Soc. 81 (1981), 359-364. MR 0597640 Zbl 0477.15007

[17] J. Rappaz, J. Sanchez Hubert, E. Sanchez Palencia, and D. Vassiliev, On spectral pollution in the finite element approximation of thin elastic "membrane" shell. Numer. Math. $\mathbf{7 5}$ (1997), 473-500. MR 1431212 Zbl 0874.73066

[18] F. Riesz and B. Sz.-Nagy, Functional analysis. Translated from the second French edition by Leo F. Boron. Reprint of the 1955 original. Dover Publications, New York, 1990. MR $1068530 \mathrm{Zbl} 0732.47001$

[19] E. Shargorodsky, Geometry of higher order relative spectra and projection methods. J. Operator Theory 44 (2000), 43-62. MR 1774693 Zbl 0994.47003

[20] E. Shargorodsky, On some open problems in spectral theory. In M. Levitin and D. Vassiliev (eds.), Operator theory and its applications. In memory of V. B. Lidskii (1924-2008). Society Translations, Series 2, 231. Advances in the Mathematical Sciences 65. Amer. Math. Soc., Providence, R.I, 2010, 173-180. MR 2757530 Zbl 1217.47002

[21] M. Strauss, Quadratic projection methods for approximating the spectrum of self-adjoint operators. IMA J. Numer. Anal. 31 (2011), 40-60. MR 2755936 Zbl 1214.65026 
Received Mach 27, 2012; revised April 11, 2012

Eugene Shargorodsky Department of Mathematics, King's College London, Strand, London WC2R 2LS, U.K.

E-mail: eugene.shargorodsky@kcl.ac.uk 